\title{
Acute bioenergetic intervention or pharmacological preconditioning protects neuron against ischemic injury
}

\author{
February 2, $2013 \cdot$ Volume $6 \cdot$ Original Research \\ Shimin Liu ${ }^{1}$, Gehua Zhen², Rung-chi Li² ${ }^{2}$ Sylvain Doré2 \\ 1 Department of Neurology, School of Medicine, Boston University, Boston, Massachusetts, USA \\ 2 Department of Anesthesiology/Critical Care Medicine, Johns Hopkins University School of Medicine, Baltimore, Maryland, USA
}

Article citation: Liu S, Zhen G, Li R, Doré S. Acute bioenergetic intervention or pharmacological preconditioning protects neuron against ischemic injury: Acute bioenergetic intervention or pharmacological preconditioning .J Exp Stroke Transl Med 2013 Feb 2, Volume 6. pp 7-17. Online access at http://www.aestm.com/article/chronic-treatment-with-flavanol-enriched-cocoa-extract-protects-against-neuronal-injury-in-forebrainischemia/

Correspondence should be sent to:Shimin Liu, MD, PhD Assistant Professor Department of Neurology Boston University School of Medicine 72 E Concord St, Evans Rm 337, Boston, MA02118 Tel: 617-638-7776; Fax:617-638-5354 Email: lius@bu.edu

\section{Abstract}

Although acute ischemic stroke has high mortality and morbidity rate but yet still has very limited treatment. In this study we have tested the concept of neuron protection by acute bioenergetic intervention or by pharmacological preconditioning with natural antioxidants. Adenosine triphosphate (ATP), pentobarbital, and suramin were encapsulated in $\mathrm{pH}$-sensitive liposomes and used as bioenergy stabilizer. We induced ATP depletion model by incubating cells with media added with ATP-depleting agents for 2 hours. Treatment with bioenergy stabilizer started 10-min post inducing of ATP-depletion. The acute treatment with bioenergy stabilizer significantly increased cell viability in neuro-2a cells. In searching for a pharmacological preconditioning candidate for reducing ischemic injury, we tested cocoa-derived flavanols using bilateral common carotid artery occlusion (BCCAO). We pretreated mice with cocoa-derived flavanols $(75 \mathrm{mg} / \mathrm{kg})$ or water orally for 7 days and subjected mice for 12 minutes BCCAO. At 7 days post-ischemia, the number of surviving hippocampal CA1 neurons was significantly higher in the treated mice than in the watertreated controls. The protection from cocoa-derived flavanols was found associated with increased total antioxidant capacity in the brain. Our results indicate that for reducing acute ischemic injury bioenergetic intervention using advanced drug delivery tools is conceptually feasible, and for reducing reperfusion related secondary injury pharmacological preconditioning may provide significant protection.

\section{Introduction}

Acute ischemic stroke is one of the leading causes of mortality and disability in developed countries, yet current treatment and prevention options are inadequate. Recombinant tissue plasminogen activator (rt-PA or alteplase) treatment for acute stroke must be given within 3-4.5 hours of onset (Saver, Albers et al. 2009) and due to narrow therapeutic window it is available to only 2-3\% of all acute ischemic stroke patients (Weintraub 2006). The majority of stroke patents are left without a specific treatment. On the other hand, the prevention of ischemic stroke is in a similar situation. Patients who have experienced transient ischemic attacks (TIAs) or previous strokes carry a risk of recurrent strokes between $5 \%$ and $20 \%$ per year (Grau, Reis et al. 2001; Weimar, Kraywinkel et al. 2002). Aspirin, with or without dipyridamole, can be effective for secondary prevention of ischemic strokes in such patients, with up to $18 \%$ or $37 \%$ respective reduction in stroke risk (Diener, Cunha et al. 1996). However, the majority of these high-risk patients will still suffer ischemic strokes despite being treated with anti-platelet medications, and TIA itself may not be able to induce ischemic preconditioning in humans. (Johnston 2004)

Searching for an effective protective method against ischemic injury is a challenging task. A systemic review has estimated that over a 1000 experimental treatments have been tested so far with little to no success.(O'Collins, Macleod et al. 2006) Novel concepts in neuroprotection for ischemic injury are urgently needed. We have developed a drug delivery tool using nanoliposome technologies that only release drug when environmental $\mathrm{pH}$ has reached a specific threshold. In this study we encapsulated adenosine triphosphate (ATP), pentobarbital, and suramin (APS) into $\mathrm{pH}$-sensitive liposomes and used it as an energy stabilizer to protect against ATP-depleting agents-induced cell injury. In the APS formula, the ATP provides direct energy, pentobarbital inhibits energy consumption, and suramin reduces ATP induced toxicity when the liposme leaks in extracellular space. The results show the APS formula is well-tolerated in cell culture, and protects neuro-2a cells from injuries induced by ATPdepleting agents.

In addition to aspirin, or like, for stroke prevention, high-risk patients also need to take a medication or a dietary component that can reduce injury from ischemic stroke that aspirin fails to prevent. Polyphenols have been shown to have neuroprotective effects in transient focal cerebral ischemia and forebrain ischemia (Simonyi, Wang et al. 2005), To test the potential of flavanol-enriched cocoa extract as a candidate for pharmacological preconditioning, we pretreated mice with cocoa extract for a week and induced bilateral common carotid artery occlusion (BCCAO) model. We found that pretreatment with the cocoa extract reduced hippocampal neuronal loss, indicating that flavanol-enriched cocoa extract may be used for reducing injury from acute ischemic strokes.

\section{Materials and methods}

2.1 Preparation of $\mathrm{pH}$-sensitive liposome.

Construction of liposome suspension. We constructed $\mathrm{pH}$-sensitive liposomes using dioleoylphosphatidylethanolamine (DOPE) and cholesteryl hemisuccinate (CHEMS), polyethylene glycol-phosphatidylethanolamine (PEG-PE) with a molecular DOPE:CHEMS:PEG-PE = 7:1:0-3\%. Chloroform solutions of lipids were mixed in glass tubes, and the solvent was evaporated through a vacuum pump to form a film of lipid layer. The lipid films were hydrated with $1 \mathrm{ml}$ content solution in a flask, which was filled with argon gas and sealed. The lipid film was gradually hydrated by gently shaking the flask at $40^{\circ} \mathrm{C}$ for $30 \mathrm{~min}$. The liposome suspension was subjected to five freezing-thawing cycles, by freezing the suspension in the freezer at $-80^{\circ} \mathrm{C}$ and then thawing the suspension in a water bath at $30^{\circ} \mathrm{C}$ (Liang, Levchenko et al. 2004). The suspension was filtered against Online access at http://nww.aestm.com/?p=817 

two stacks $800,600,400$, and $200 \mathrm{~nm}$ pore size polycarbonate filters using a hand-held extruder (Avanti Polar Lipids, Alabaster, AL). This process yields liposomes with a size distribution around $150 \mathrm{~nm}$. For preparing small-sized liposomes (Yamaguchi, Nomura et al. 2009), liposome dispersion was placed in a bath sonicator (Laboratory Supplies, Hicksville, NY) for $5-10$ minutes at $0{ }^{\circ} \mathrm{C}$ to produce clear solution, and then be extruded (5 times for each pore size) through two stacks $100,80,50$, or $30 \mathrm{~nm}$ pore size polycarbonate filters. Plain liposomes were constructed using same volume of $5 \mathrm{mM}$ HEPES buffered saline.

"Energy formula"-loaded liposomes. The formula consisted of 90 umole ATP, 80 umole pentobarbital, and $7 \mu$ mole suramin in $1 \mathrm{ml}$ solution of $5 \mathrm{mM}$ HEPES buffer ( $\mathrm{pH}$ was adjusted to 7.4 with $1 \mathrm{MNaOH}$ ). The total content (ATP, pentobarbital, suramin) to total lipid (liposome) molar ratio was 5 to 1.

Purification of DSPE-PEG coated $\mathrm{pH}$-sensitive nanoliposomes. We used the centrifugation method to achieve highly purified nanoliposomes. $100 \mu \mathrm{l}$ of raw suspension of "energy formula"-loaded nanoliposomes was diluted to $1 \mathrm{ml}$ with HEPES-buffered saline (HBS), pH 7.4 and centrifuged in an Optima TXL ultracentrifuge (Beckman) for 20 -min at $10,000 \mathrm{~g}$ at $15^{\circ} \mathrm{C}$. After the supernatant had been removed, the residual liposomal pellets were resuspended in $1 \mathrm{ml}$ of HBS and the centrifugation was carried out again as described above. This cycle was repeated three times. The final liposomal pellets were resuspended with $1 \mathrm{ml}$ of HBS and dialyzed for 24 hours against HBS through a Spectra dialysis membrane with molecular weight cutoff at 2000 (Spectrum Laboratory).

$\mathrm{pH}$-sensitivity of "energy formula"-loaded nanoliposomes in buffers. One microliter of "energy formula"-loaded nanoliposomes (final phospholipid concentration between 5 and $6 \mu \mathrm{M})$ was added to $2 \mathrm{ml}$ of MES-buffered saline $(140 \mathrm{mM} \mathrm{NaCl}, 10 \mathrm{mM} \mathrm{MES})$ and incubate for 1 - $\mathrm{h}$ at various pH values: $5.5,6,6.5,6.8,7.0$, and 7.4. Before and after incubation, the solution was sampled by taking a $0.10 \mathrm{ml}$ aliquot and diluting it in $1.9 \mathrm{ml}$ of MES-buffered saline, $\mathrm{pH} 7.4$. The total release of encapsulated contents was achieved by the addition of Triton $\mathrm{X}-100$ (final concentration $0.1 \%$ ). ATP concentration in sample were measured using a Biovision ATP assay kit by following manuracturer's instruction. The percentage of content release under a specific $\mathrm{pH}$ condition was calculated according to the formula: percent leakage $=((\mathrm{QpH}-\mathrm{Q} 0) /(\mathrm{Q} 100-\mathrm{Q} 0)) \mathrm{x} 100$, where $\mathrm{Q} 0$ is the quantity at neutral $\mathrm{pH}, \mathrm{QpH}$ is the release quantity after incubation under a specific $\mathrm{pH}$ condition without the addition of Triton X-100, and $\mathrm{Q} 100$ is the totally released contents after adding X-100.

\subsection{Testing acute bioenergetic intervention in cell culture system Neuro-2a cell line.}

Neuro-2a cell line. Neuro-2a cell culture and differentiation. Neuro-2a cell line was purchased from ATCC (catalog \# CCL-131) and cultured in Dulbecco's modified Eagle's medium supplemented with $10 \%(\mathrm{v} / \mathrm{v}$ ) fetal bovine serum, $100 \mathrm{U} / \mathrm{ml}$ penicillin, $100 \mu \mathrm{g} / \mathrm{ml}$ streptomycin, and $292 \mu \mathrm{g} / \mathrm{ml}$ glutamine at $37^{\circ} \mathrm{C}$ in a humidified $5 \%$ (v/v) CO2 incubator. The cells were seeded at $1.0 \times 106$ per cm2, and then incubated for $24 \mathrm{~h}$ in $90-\mathrm{mm}-$ diameter plastic dishes. Induction of differentiation was performed by changing media to $2 \%$ or $0.5 \%$ (v/v) fetal bovine serum with or without $10-20$ $\mu \mathrm{M}$ retinoic acid (Sigma) for $96 \mathrm{~h}$. The culture medium was routinely changed every 2 days. (Shinjyo and Kita 2006; Tremblay, Sikorska et al. 2010)

Rat astrocytes and Growth Medium were purchased from Cell Applications, Inc. Cells at a density of $5.0 \times 105$ cells/ml were plated in T75 flasks containing $15 \mathrm{ml}$ of Rat Astrocyte Growth Medium, cultured in in a $37^{\circ} \mathrm{C}, 5 \% \mathrm{CO} 2$ humidified incubator, and change Rat Astrocyte Growth Medium every other day until the cells reach $60 \%$ confluent. Subculturing the cells were done when the RA reach $80 \%$ confluent and seeded at density of 6,000 cells per $\mathrm{cm}^{2}$

Rat Brain Microvascular Endothelial Cells (RBMVEC) were purchased from Cell Applications, Inc. We followed General Instructions for Culturing that came with purchased cells from the company for RBMVEC culture. Briefly, T-75 flasks were coated with 7.5 ml of Attachment Factor Solution for 30 minutes at $37^{\circ} \mathrm{C}$, and then the coated flask was loaded with $15 \mathrm{ml}$ of Growth Medium. Cryopreserved vial of RBMVEC Cells at a density of $5.0 \times 10^{5}$ cells $/ \mathrm{ml}$ were thawed in a $37^{\circ} \mathrm{C}$ water bath for 1 minute, and seeded into the coated T-75 flask containing 15 ml of RBMVEC Growth Medium, and cultured in a $37^{\circ} \mathrm{C}, 5 \% \mathrm{CO} 2$ humidified incubator, change to fresh RBMVEC Growth Medium after 24 hours and every other day until the cells reach $60 \%$ confluent. Subculture were done when the RBMVEC reach $80 \%$ confluent.

Cell toxicity of "Energy formula"-loaded liposomes. Some liposomes may release ATP into extracellular space which is toxic to cells. The suramin component in the formula was designed to reduce the toxicity induced by extracellular ATP. Liposome suspension was added to culture media with a volume ratio of 1 to 25 . Neuro-2a cells were co-cultured with plain liposome, 3-component energy formula or 2-component energy formula missing suramin for 2 hours and followed by cell viability assay.

ATP depletion model and treatment by energy formula-loaded liposome suspension. Cells were induced graded ATP depletion (Lieberthal, Menza et al. 1998) by change to media that contain 2-deoxyglucose (DOG) (5 mM, an inhibitor of glycolysis), $1 \mu \mathrm{M}$ rotenone (inhibitor of complex I), and antimycin $(2 \mu \mathrm{M}$, inhibitor of mitochondrial electron transport chain between cytochrome b and c). At 10-min post inducing ATP depletion, liposome suspension was added to media with a liposome to media ratio of 1:10, the final concentrations were 9 mM ATP, 8 mM pentobarbital, $0.7 \mathrm{mM}$ suramin, and $3.5 \mathrm{mM}$ lipids.

Cell viability assay. Cell viability was measured by the 3-(4, 5-dimethylthiazol-2-yl) - 2, 5-diphenyltetrazolium bromide (MTT) assay after 1-h ATP depletion. MTT was added to the cultures to a final concentration of $375 \mu \mathrm{g} / \mathrm{ml}$. The insoluble purple formazan product was dissolved with a buffer ( $20 \%$ SDS and $50 \%$ N, N-dimethylformamide (DMF)) after 2-4 h of incubation with MTT. Optical density was read at 580 nm, and the percentage of cell survival was calculated relative to control cells without ATP depletion. (Chang, Jiang et al. 2008)

\subsection{Pharmacological preconditioning against ischemic injury.}

Animal care and group design. This study was performed in accordance with the National Institutes of Health guidelines for the use of experimental animals. Protocols were approved by the Johns Hopkins Institutional Animal Care and Use Committee. For in vivo studies, we used adult C57BL/6 mice weighing 22-25 g. Mice were randomized into different groups. Quantification of cocoa induced neuroprotection was conducted in chronic treatment regimens of cocoa or water ( $n=20$ per group). Additional 12 mice were set aside as sham operated group for obtaining baseline neuronal counting, In another experiment, cocoa-induced changes in total antioxidant capacity (TAC) and glutathione (GSH) level were studied ( $n=$ 4 per group).; Immunohistochemical staining, which was used to quantify astrocytes, microglia, and 8-hydroxydeoxyguanosine (8-OHdG), was studied in water or cocoa treated mice, with $n=7$ in each group.

Administration of the cocoa extract. Many cocoa extracts are available on the market. The optimal selection of cocoa extract is critical for neuroprotective studies because the manufacturing process can substantially affect the amount of active content (Romero-Perez, LamuelaRaventos et al. 2001). We chose the extract manufactured by Symbioscience (Gaithersburg, MD) because it provides a highly standardized and characterized product that is enriched in flavanol content. The cocoa powder was dissolved in distilled water to a final concentration of $3 \mathrm{mg} / \mathrm{ml}$. Chronic pretreatment in mice was accomplished by giving cocoa solution at a daily dose of $75 \mathrm{mg} / \mathrm{kg}$ body weight via gavage for 7 days. Control groups received the same volume of water vehicle. Mice in cocoa or water treated group were coded for purpose of concealment during subsequent observational process. Group code was revealed after analyses had been completed. 
Induction of transient forebrain ischemia. Transient forebrain ischemia was induced on day 8 after initiation of cocoa treatment as described previously by Zhen et al (Zhen and Dore 2007) with minor modification. The anesthetic and surgical environmental settings were predetermined through a pilot experiment in which maintenance of blood pressure, blood gas, and core temperature within normal ranges was shown to be the optimal configuration. Mice were anesthetized with isoflurane ( $4 \%$ for induction and $1.5 \%$ for maintenance). A vertical midline incision was made in the neck, and then the trachea was exposed and intubated. Throughout the procedure, the mice were mechanically ventilated. Bilateral common carotid arteries were exposed and occluded for $12 \mathrm{~min}$. Blood flow was monitored by DRT4 laser-Doppler flowmetry (Moor Instruments, Wilmington, DE). A reduction of at least $90 \%$ from baseline in the first $2.5 \mathrm{~min}$ was considered to be a successful forebrain ischemia and was used for analyses (Yonekura, Kawahara et al. 2004). After BCCAO, mice were kept in a temperature-controlled chamber at $340 \mathrm{C}$ for $24 \mathrm{~h}$ to allow for full recovery of body temperature regulation. Sham groups were subjected to a similar procedure, but the carotid arteries were not occluded. Mice were allowed to survive for 7 days after the surgery.

Quantification of hippocampal injury in forebrain ischemia model. On day 7 after BCCAO, mice were deeply anesthetized and transcardially perfused with $50 \mathrm{ml}$ heparinized normal saline followed by $50 \mathrm{ml}$ of $4 \%$ paraformaldehyde. Then they were transcardially infused with $1 \mathrm{ml}$ latex to allow visualization of the posterior communicating artery (PComA) under a microscope (Zhen and Dore 2007). Mice were subgrouped as PComAabsent if the PComA diameter was less than $1 / 3$ of normal size, which is similar to the posterior cerebral artery, or as PComA-present if the PComA diameter was greater than $1 / 3$ of normal size. After the documentation of PComA patency, brain blocks were post-fixed for $24 \mathrm{~h}$ by immersion in $4 \%$ paraformaldehyde in phosphate-buffered saline for paraffin embedding. Paraffin-embedded brain blocks were sectioned at 10 um thickness and then stained with hemotoxylin and eosin to allow for counting of live neurons in the CA1 sector. Three representative sections were selected from each mouse. The number of surviving neurons in each mouse was obtained by averaging the numbers counted from these selected slides and was expressed as the number of viable neurons per linear millimeter. Because consistent hippocampal injury was only seen in PComA-absent mice, therefore, only these mice were included for final analysis (Zhen and Dore 2007).

Measurement of total antioxidant capacity (TAC) and glutathione (GSH) in brain tissue. Mice were gavaged with cocoa or vehicle as described above. At $0 \mathrm{~h}$ and 7 days after initiation of treatment, mice were killed with an overdose of pentobarbital and perfused with 50 ml of heparinized normal saline. Brains were harvested to measure TAC and GSH. We used the ApoGSH Glutathione Colorimetric Detection Kit (K261-100, BioVision, Mountain View, CA) and Total Antioxidant Capacity Assay Kit (K274-100, BioVision) according to the manufacturer's instructions.

Immunohistochemical staining was carried out according to the standard procedures described in the manufacturer's instructions. Briefly, paraffin sections were deparaffinized, hydrolyzed, and washed in tris-buffered saline. Nonspecific binding was blocked with $4 \%$ normal horse serum in trisbuffered saline for $1 \mathrm{~h}$. Then sections were incubated with primary antibodies at $4^{\circ} \mathrm{C}$ overnight. Antibodies against GFAP, Iba1, and $8-\mathrm{OHdG}$, were used to label reactive astrocytes, microglia, and free radical-induced DNA damage, respectively. The immunoperoxidase method with diaminobenzidine chromogen (ABC Elite, Vector Laboratories, Burlingame, CA) was used to visualize primary antibody staining. Digital images were acquired through a Nikon light microscope (TE2000-E) equipped with a Nikon camera and Nikon ACT-1 software (Nikon, Melville, NY). For quantification, three representative regions were selected under 40x objective lens from each section. A total of three sections were selected from each mouse. The percentage of positively immunostained areas or cell number per $40 x$ objective field was calculated with Adobe Photoshop 7.0 (San Jose, CA)

\subsection{Statistics}

Data are expressed as the mean \pm standard deviation (SD). Differences between means were analyzed by Student's t-test for comparisons between two groups and by one-way analysis of variance (ANOVA) for multiple group comparisons, followed by Scheffe posthoc multiple comparisons. Statistical significance was set at $\mathrm{P}<0.05$

\section{Results}

3.1 PEGylated pH-sensitive liposome system

As shown in Figure1, liposome suspension incorporated 3\% PEG 34000 has maximal release range from pH 6.5 to 7.0 , which is more closer to the reported penumbral intracellular pH (Anderson et al 1999). Liposome without PEG has a high background release (data not shown). 


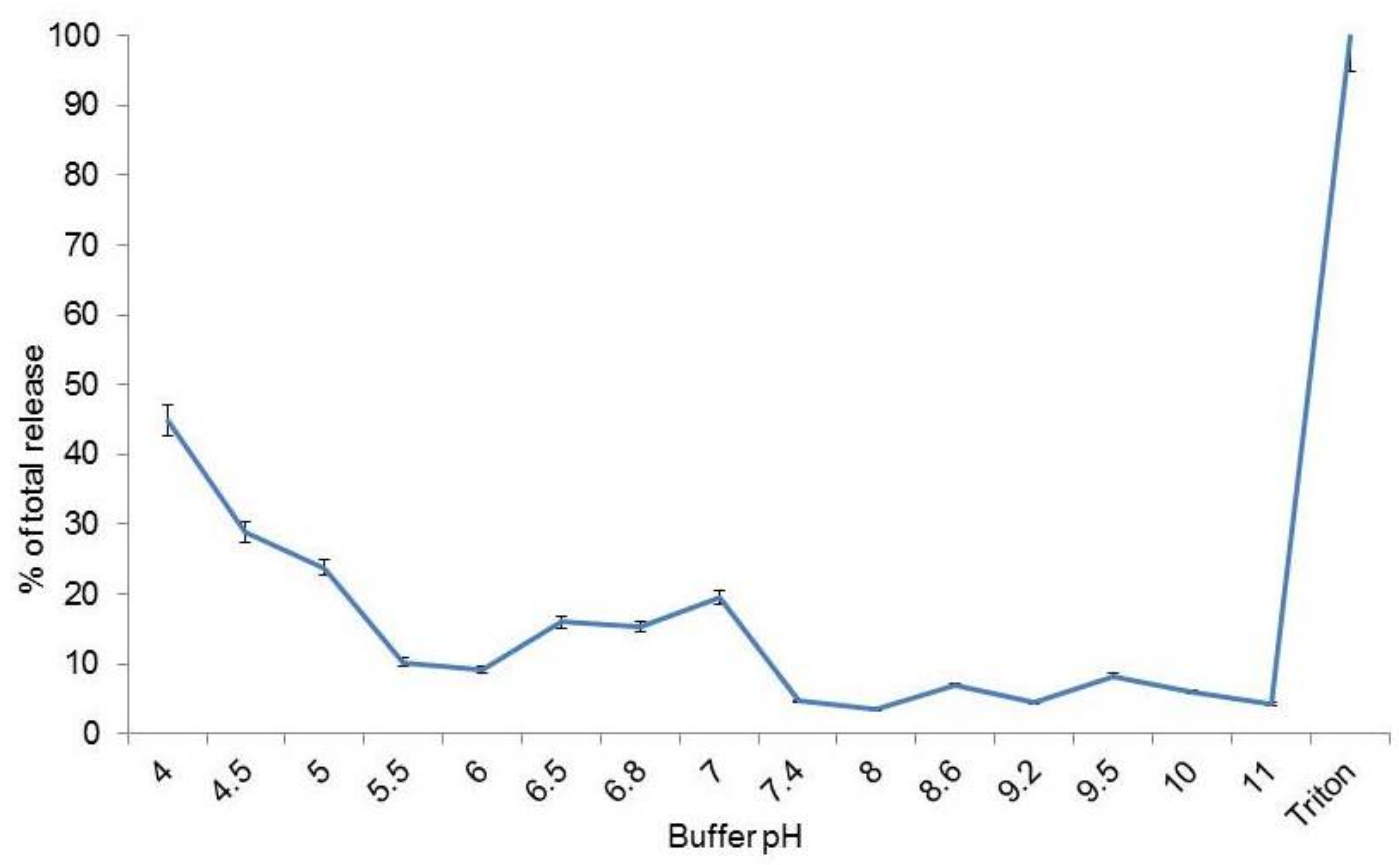

Figure 1.

$\mathrm{pH}$-triggered drug release. We constructed $\mathrm{pH}$-sensitive liposomes using dioleoylphosphatidylethano-lamine (DOPE) and cholesteryl hemisuccinate (CHEMS), with a molecular ratio DOPE:CHEMS:PEG-PE = 7:1:0-3\%. Using PEG 34000 we achieved a maximal release $\mathrm{pH}$ range from $\mathrm{pH} 7$ to $\mathrm{pH} 6.8$.

3.2 Acute bioenergetic intervention against ATP-depleting injury.

The "energized" pH-sensitive liposomes reduce neuronal injury in ATP-depletion model. We induced ATP depletion model for 2 hours. Treatment with "energy formula"-loaded $\mathrm{pH}$-sensitive liposomes started 10-min post inducing of ATP-depletion. The treatment significantly increased cell viability in neuro-2a cells (Figure 2). A similar trend was also found in endothelial cells and astrocytes, but the ATP-depletion caused damage maybe too severe in these cells and the therapy didn't reach significance.

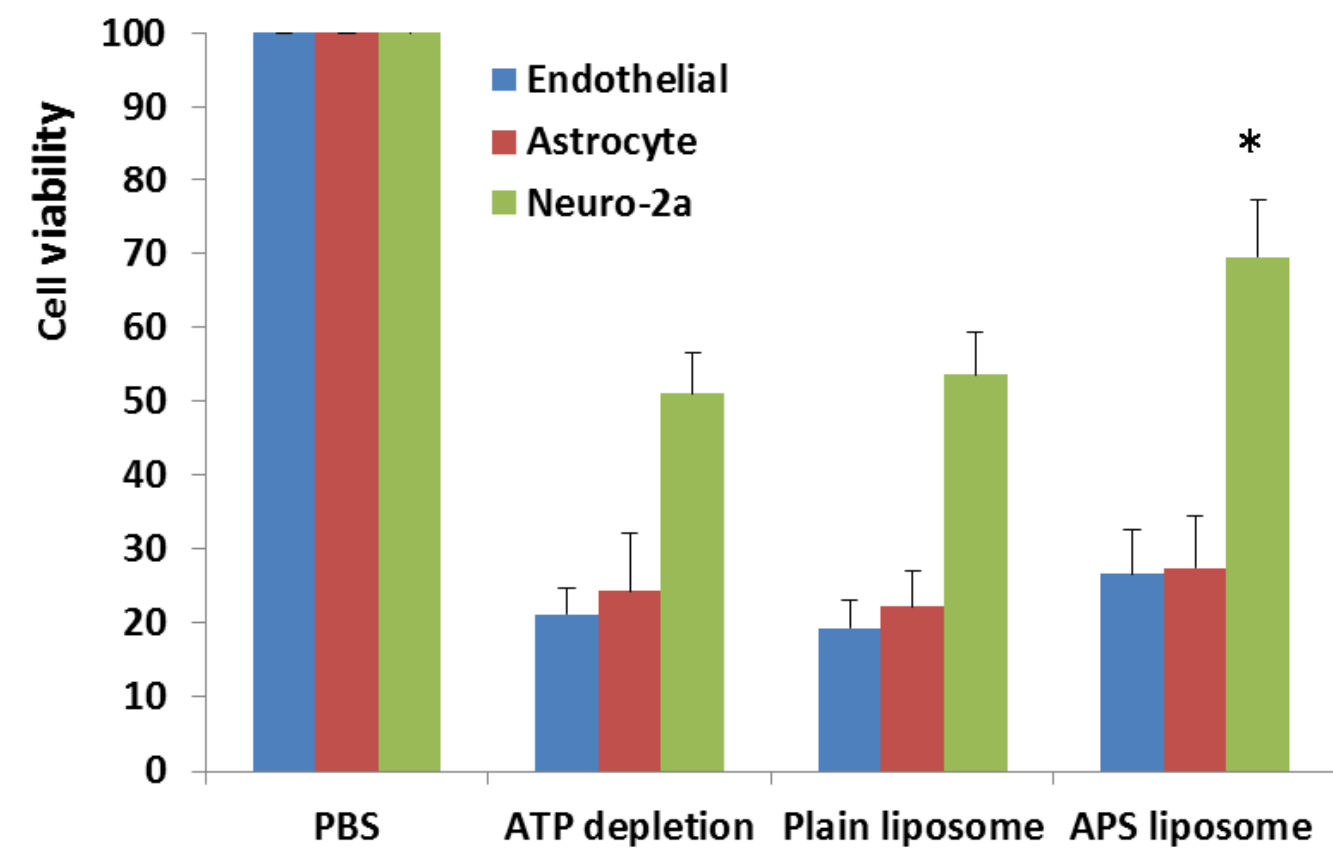

Figure 2.

The three component energy formula protected neuro-2a cells against ATP-depletion-induced injury. We induced ATP depletion model by incubating cells with media added with $5 \mathrm{mM}$ 2-Deoxy-D-glucose, $1 \mu \mathrm{M}$ Rotenone, $2 \mu \mathrm{M}$ antimycin A for 2 hours. Treatment with "energy formula"-loaded $\mathrm{pH}$-sensitive liposomes $(80 \mu \mathrm{g}$ lipids $/ \mathrm{ml}$ media) started 10-min post inducing of ATPdepletion. The "energy formula" that we used in this experiment contains $90 \mathrm{mM}$ ATP, $80 \mathrm{mM}$ pentobarbital, and $7 \mathrm{mM}$ suramin Online access at http://www.aestm.com/?p=817 
Suramin in "energized" pH-sensitive liposomes effectively reduces the adverse effect of extracellular ATP. We further tested the toxicity of the "energy formula" that we used for constructing $\mathrm{pH}$-sensitive liposomes. The formula solution was added to culture media with a ratio of 1 to 25 and cultured with cells for 2 hours. Suramin is a non-selective P2 receptor antagonist (Kharlamov et al 2002; Millart et al 2009), which is used to minimize the adverse effects caused by ATP binding of P2 receptor. DPBS and the formula without suramin were used as a control. Cell viability was measured by MTT assay. It showed that the 3-component formula (APS in the right figure) is well-tolerated by all three cell types when added to culture media (Figure 3.).

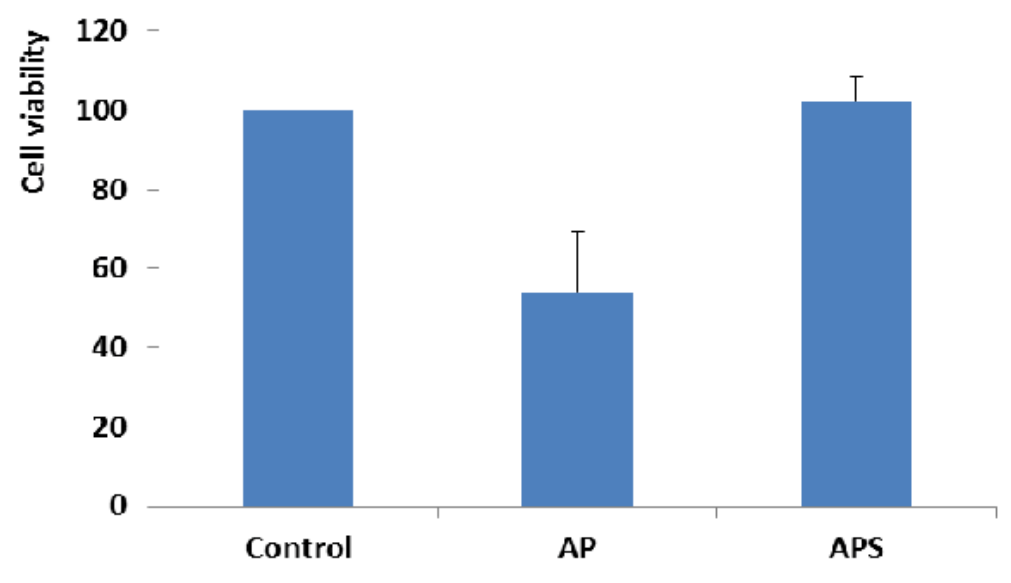

\section{Figure 3.}

Suramin eliminated ATP-induced cell toxicity. We further tested the toxicity of the "energy formula" that we used for constructing $\mathrm{pH}$-sensitive liposomes. The formula solution was added to culture media with a ratio of 1 to 25 and cultured with cells for 2 hours. DPBS and the formula without suramin were used as a control. Cell viability was measured by MTT assay. It showed that the 3-component formula (APS in the right figure) is well-tolerated by all three cell types when added to culture media.

\subsection{Pharmacological preconditioning against ischemic injury}

$\mathrm{BCCAO}$ and quality control. During $\mathrm{BCCAO}$, the monitored rectal temperature was in the range of $37.5 \pm 0.5^{\circ} \mathrm{C}$. The mortality rate of the group that received 7 days of pretreatment with cocoa was $10 \%(2 / 20)$ and was similar to that in the water-treated control group (15\%, $3 / 20)$. No animal died in other groups. The absence of PComAs correlated well with the hippocampal neuronal damage in transient forebrain ischemia, a finding that is supported by our previous results (Zhen and Dore 2007). Therefore, for the immunostaining and subsequent quantification, we used only hemispheres that did not show a patent PComA. In the experiment of quantification of hippocampal injury, 9 (of 17) and 11 (of 18) survived mice were graded as PComA-absent in water treated and cocoa treated groups, respectively. In the experiment of immunohistochemical staining, 4 (of 7) and 5 (of 7 ) mice were graded as PComA-absent in water treated and cocoa treated groups, respectively.

Chronic cocoa pretreatment preserved CA1 neurons in forebrain ischemia. Seven days of cocoa pretreatment significantly increased the number of surviving neurons in the hippocampal CA1 sectors as compared to that in the control group (Figure 4A-4D). In the water-treated group, the number of surviving neurons was $58.5 \pm 57.0 /$ linear $\mathrm{mm}$; chronic cocoa pretreatment significantly increased that number to $124.9 \pm 76.0 /$ inear mm $(P=0.038 ;$ Figure 4E). The average neuronal density in sham-operated mice was $249.8 \pm 16.1 /$ linear mm. 

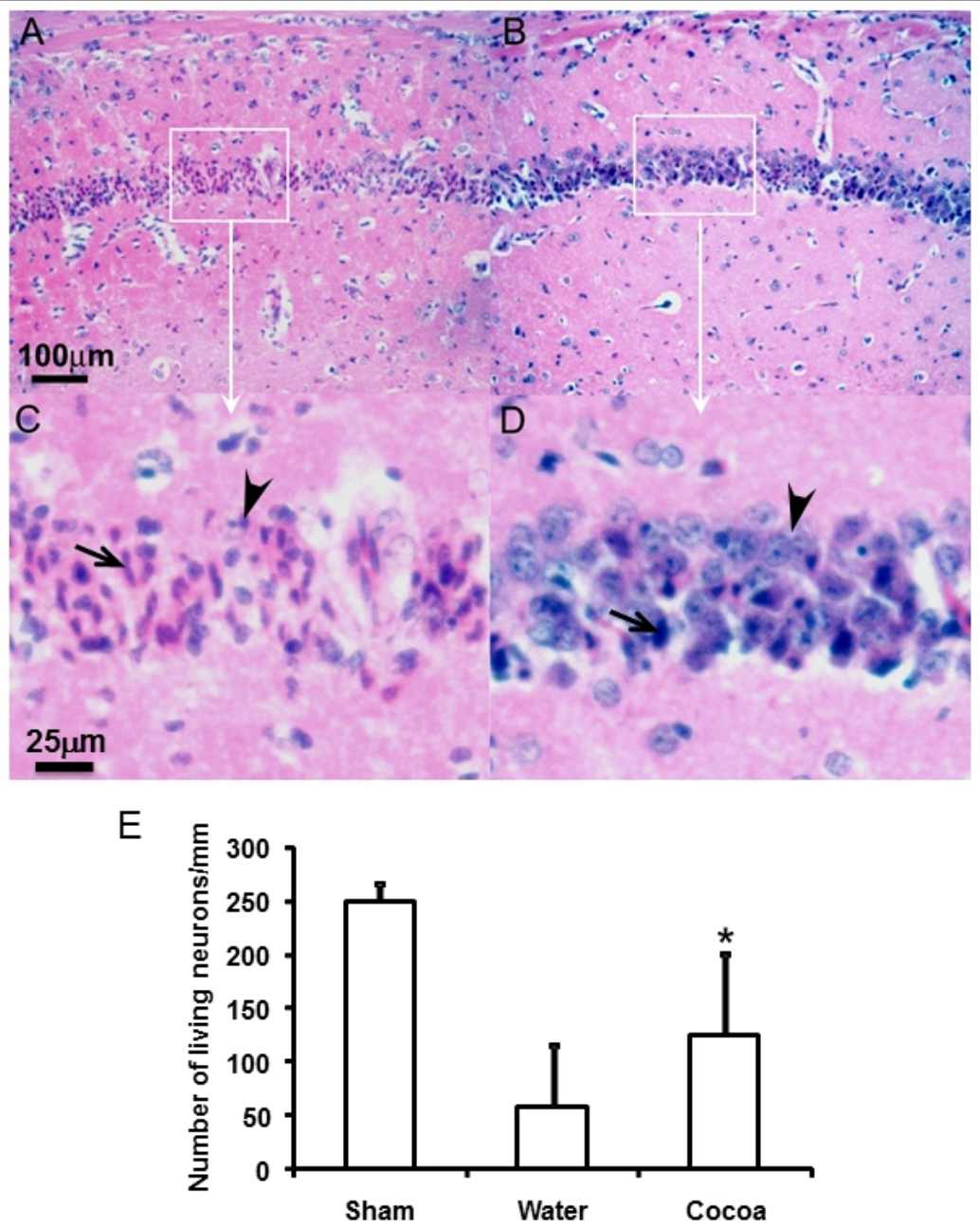

Figure 4.

Numbers of living neurons in hippocampal CA1 sectors. Mice were administered water $(A)$ or cocoa extract $(75 \mathrm{mg} / \mathrm{kg}$ per day; B) for 7 days before being subjected to 12-min bilateral common carotid artery occlusion. Brains were harvested after 7 days, sectioned, and stained with hemotoxylin and eosin. (C) Higher magnification of A; (D) higher magnification of B. Arrows, dead neurons; arrowheads, live neurons. (E) Quantification of living neurons in the CA1 sector for the sham $(n=12)$, water $(n=9)$, and cocoa-treated $(n=11)$ groups. Data are expressed as mean \pm SD. Cocoa treatment significantly preserved CA1 neurons compared with the water-treated group. ${ }^{*} \mathrm{P}<0.05$ compared with the other groups (ANOVA plus Scheffe multiple comparison). Only hemispheres without PComAs were included in data analyses.

Chronic cocoa treatment reduced GSH but preserved TAC. To further explore the mechanism by which chronic pretreatment with cocoa extract might afford protection, we measured TAC and GSH levels in brain tissue. We observed that brain TAC (Figure 5A) and GSH (Figure 5B) levels were significantly increased after 7 days of treatment compared with baseline $(P<0.05$ by t-test). 
A

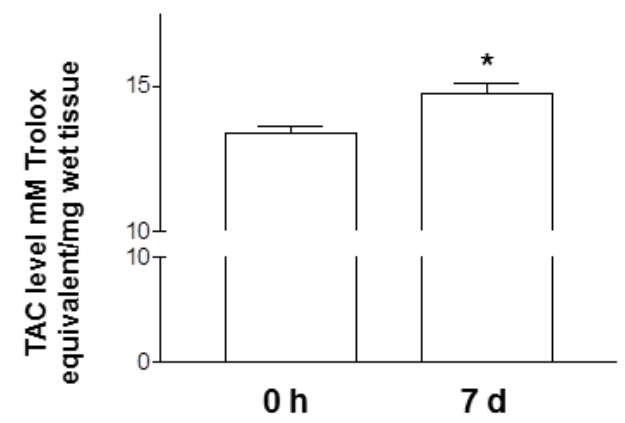

$\mathrm{B}$

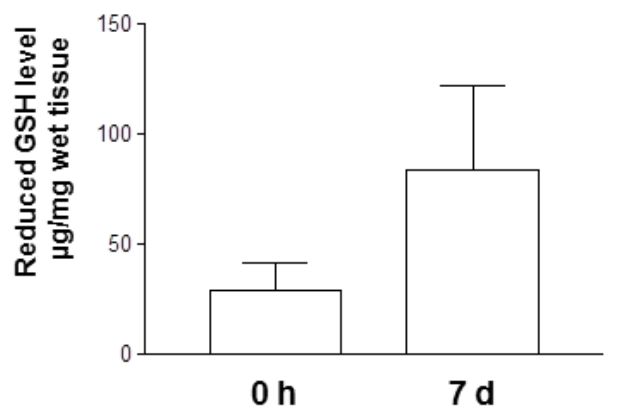

\section{Figure 5.}

Total antioxidant capacity (TAC, A) and glutathione (GSH, B) levels in brain tissue at different time points after administration of cocoa extract. Data are expressed as mean $\pm \mathrm{SD} ;{ }^{*} \mathrm{P}<0.05$ compared with $0 \mathrm{~h}$ by ANOVA and Scheffe multiple comparison; ns, not significant; $n=4$ per group.

Glia reactivity was reduced in cocoa-treated mice. The reactivity of both microglia and astrocytes in the hippocampal region at 7 days after forebrain ischemia was less severe in the cocoa-treated group than in the control group (Figure 6). Reactive astrocytes (Figure 6A and 6B) were larger, as shown by anti-GFAP staining and through the comparison of positive anti-GFAP stained areas (Figure 6B, and 6F). However, the total number of astrocytes was not significantly different in cocoa-treated mice (Figure 6E). In contrast, the microglia increased in both size and number (Figure 6C, 6D, 6G, and 6H). 


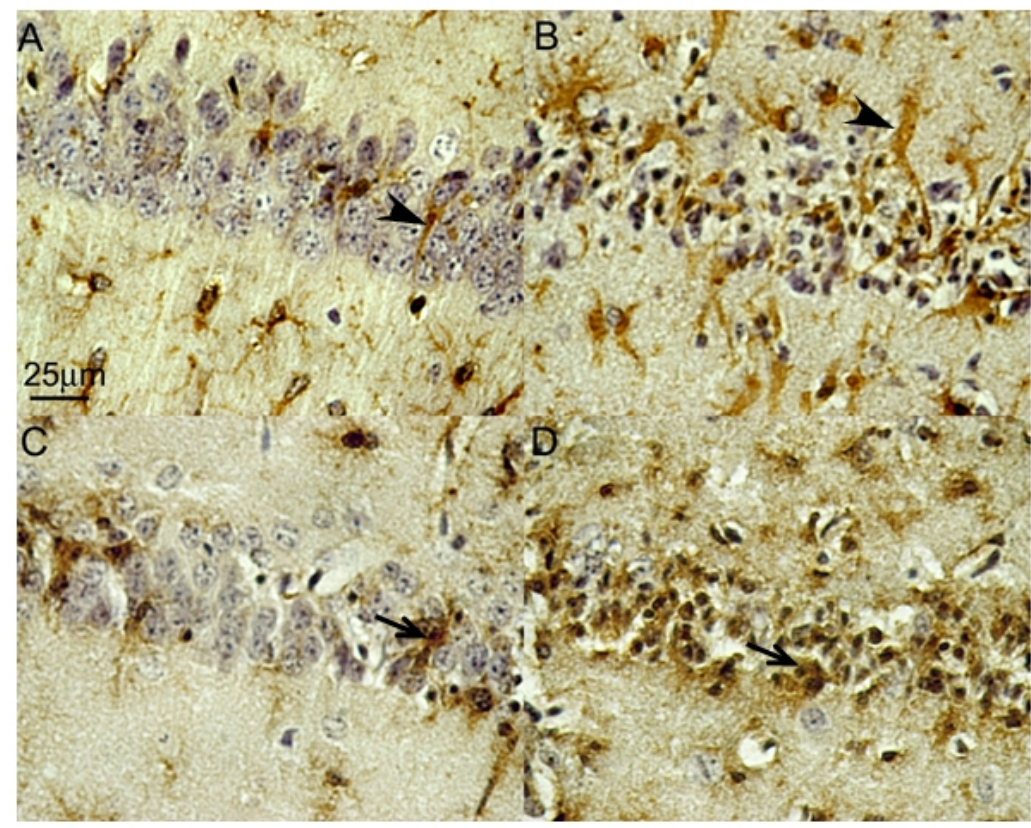

E

G

$\mathrm{H}$
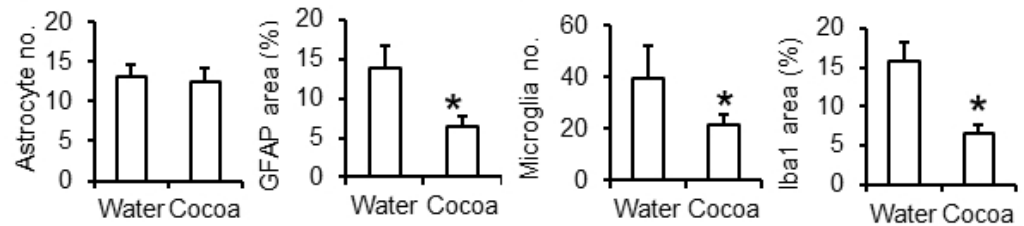

Figure 6.

Glia reactivity is lower in cocoa-treated than in water-treated mice. Mice were administered water or cocoa extract $(75 \mathrm{mg} / \mathrm{kg}$ per day) for 7 days before being subjected to 12-min bilateral common carotid artery occlusion. Brains were harvested after 7 days and sectioned for staining. Anti-GFAP staining of brains from (A) cocoa-treated and (B) water-treated mice; astrocytes are indicated by arrowheads. Anti-lba1 staining of brains from (C) cocoa-treated and (D) water-treated mice; microglia are indicated by arrows. ( $E$ and F) Quantification of reactive astrocytes; ( $G$ and $H$ ) quantification of microglia. Data are expressed as mean $\pm S D$; cell number is expressed per $40 x$ objective field. $n=4$ for the cocoa-treated group; $n=5$ for the water-treated group; ${ }^{*} \mathrm{P}<0.05$ compared to the water-treated group by two-tailed independent Student's t-test.

Cocoa treatment reduces 8-OHdG production. The 8-OHdG was used as an indicator of free radical-induced DNA damage (Figure 7). Production of $8-\mathrm{OHdG}$ was quantified by calculating the percentage of area positively stained by anti-8-OHdG. Seven days after forebrain ischemia, the 8OHdG production was increased in the cytosol and the nucleus (Figure 7C and 7D), astrocytes, and microglia. The cocoa-treated group had significantly lower production of $8-\mathrm{OHdG}$ than did the control group, $\mathrm{P}<0.05$ (Figure 7E). 

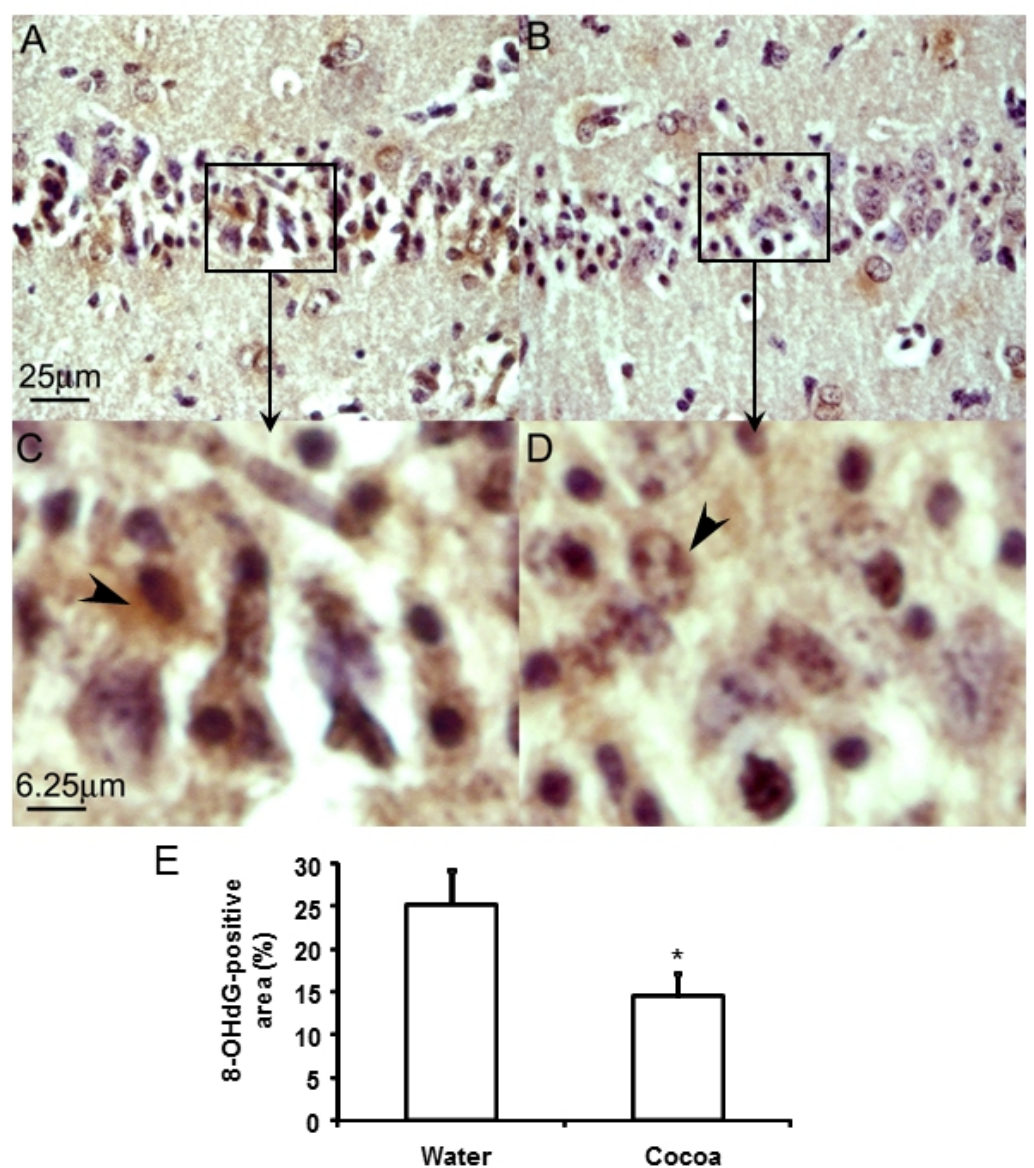

\section{Figure 7.}

Free-radical-induced DNA damage is lower in cocoa-treated than in water-treated mice. Mice were administered water (A) or cocoa extract (75 mg/kg per day; B) for 7 days before being subjected to 12-min bilateral common carotid artery occlusion. Brains were harvested after 7 days and sectioned for staining with anti-8-hydroxy-2'-deoxyguanosine (8-OHdG). (C and D) Higher magnification of $A$ and $B$, respectively; arrowheads indicate 8-OHdG-positive cells. (E) Quantification of 8-OHdGpositive area. Data are expressed as mean $\pm S D ; n=4$ for the cocoa-treated group; $n=5$ for the water-treated group; ${ }^{*}<<0.05$ compared with the water-treated group by two-tailed independent Student's t-test.

\section{Discussion}

Because stroke prevention by aspirin or dipyridamole can only reduce $18 \%$ to $37 \%$ in stroke risk (Diener, Cunha et al. 1996 ), reducing strokerelated brain injury becomes of paramount importance. This can be achieved through acute treatment after stroke has occurred, or through preconditioning approaches in high-risk patients. The ATP-depleting model used in this study produced acute injury that mimic the acute injury that occur in the narrow therapeutic window in acute ischemic stroke; in the other hand, the global ischemia model used in his study observed injury at 7-days post-ischemia, which included delayed brain injury.

Except t-PA thrombolytic treatment, the exploration of acute neuroprotective treatment for ischemic stroke was not successful. Although many reasons have been considered for the failure of stroke treatment translation, drug delivery tools and an effective treatment strategy will always be the essentials for acute ischemic stroke treatment. From this point-of-view, we explored the feasibility of using novel drug delivery system and bioenergetic intervention strategy for ischemic injury protection in cell culture system. pH-sensitive liposomes have been used in cancer therapy (Karanth and Murthy 2007) because of tumor tissue' acidic microenvironment. Ischemic tissue also has low pH, but this feature has not yet been explored for drug delivery to ischemic region. We have constructed a type of $\mathrm{pH}$-sensitive liposome that has maximal release at penumbral $\mathrm{pH}$ level. We used this liposome encapsulated ATP molecules and pentobarbital, a metabolism inhibitor for pH-sensitive delivery of bioenergetic intervention. Neuro-2a cell line is widely used in neurotoxic studies (LePage, Dickey et al. 2005) and also in oxygen/glucose deprivation (OGD) models (Mahesh, Jung et al. 2011; Chio, Lin et al. 2013). We use this model for ATP-depletion because these ATP-depleting agents are actually toxic agents to bioenergy production. Our results show such treatment is neuroprotective against acute ATP-depleting damage in cell culture system. Further optimization and in vivo experiments will be needed to test its efficiency for delivering bioenergetic intervention to ischemic tissue.

Reducing ischemic injury through increasing brain tissue's tolerance to ischemia via pharmacological preconditioning is also possible.(Bahjat, Gesuete et al. 2013) Achieving ischemic preconditioning through natural extracts is more attractive for its therapeutic value. We found that 7 days of cocoa pretreatment better preserved hippocampal neuron in mice suffered BCCAO. The number of surviving neurons in cocoa-treated mice was significantly higher than that in water-treated mice. Cocoa-induced neuroprotection was found to be associated with a significant attenuation in glia reactivity and free radical DNA damage, as demonstrated by immunohistochemical staining for GFAP, Iba1, and 8-OHdG.

It has been documented that polyphenols also have pro-oxidative properties (Park, Anrather et al. 2004; Nemeikaite-Ceniene, Imbrasaite et al. 2005; Raza and John 2005; Lee, Kundu et al. 2006; Hadi, Bhat et al. 2007; Robaszkiewicz, Balcerczyk et al. 2007; Murzakhmetova, Moldakarimov et al. 2008). Under certain conditions, polyphenols have been reported to generate reactive oxygen species (Galati, Sabzevari et al. 2002; Chan, 
Shimin Liu Acute bioenergetic intervention or pharmacological preconditioning

J Exp Stroke Transl Med (www.aestm.com) Vol 6 pp $10-11$ Galati et al. 2003). The pro-oxidative property of polyphenols may mediate their neuroprotection through a preconditioning effect, potentially by initiating an intracellular preconditioning cascade. The mechanism may be similar to another natural phenol, resveratrol, which has been found to have pharmacological preconditioning against myocardial ischemic/reperfusion injury (Imamura, Bertelli et al. 2002; Chander and Chopra 2005; Zhao, Kobori et al. 2006), or cerebral ischemic stroke (Dong, Gao et al. 2007; Tsai, Hung et al. 2007; Dong, Gao et al. 2008). Based on our results, flavanol-enriched cocoa extract could be a candidate for pharmacological preconditioning against various subtypes of strokes. Further exploration will be needed to determine how cocoa-induced changes in redox status mediate pharmacological preconditioning.

\section{References}

Anderson, R. E., W. K. Tan, et al. (1999). "Brain acidosis, cerebral blood flow, capillary bed density, and mitochondrial function in the ischemic penumbra." J Stroke Cerebrovasc Dis 8(6): 368-379.

Bahjat, F. R., R. Gesuete, et al. (2013). "Steps to translate preconditioning from basic research to the clinic." Transl Stroke Res 4(1): 89-103.

Chan, T. S., G. Galati, et al. (2003). "Simultaneous detection of the antioxidant and pro-oxidant activity of dietary polyphenolics in a peroxidase system." Free Radic Res 37(7): 787-794.

Chander, V. and K. Chopra (2005). "Role of nitric oxide in resveratrol-induced renal protective effects of ischemic preconditioning." J Vasc Surg 42(6): 1198-1205.

Chang, S., X. Jiang, et al. (2008). "Exogenous low dose hydrogen peroxide increases hypoxia-inducible factor-1alpha protein expression and induces preconditioning protection against ischemia in primary cortical neurons." Neurosci Lett 441(1): 134-138.

Chio, C. C., J. W. Lin, et al. (2013). "MicroRNA-210 targets antiapoptotic Bcl-2 expression and mediates hypoxia-induced apoptosis of neuroblastoma cells." Arch Toxicol 87(3): 459-468.

Diener, H. C., L. Cunha, et al. (1996). "European Stroke Prevention Study. 2. Dipyridamole and acetylsalicylic acid in the secondary prevention of stroke." J Neurol Sci 143(1-2): 1-13.

Dong, W., D. Gao, et al. (2008). "New insights into mechanism for the effect of resveratrol preconditioning against cerebral ischemic stroke: Possible role of matrix metalloprotease-9." Med Hypotheses 70(1): 52-55.

Dong, W., D. Gao, et al. (2007). “Mitochondria biogenesis induced by resveratrol against brain ischemic stroke.” Med Hypotheses 69(3): 700-701.

Galati, G., O. Sabzevari, et al. (2002). "Prooxidant activity and cellular effects of the phenoxyl radicals of dietary flavonoids and other polyphenolics." Toxicology 177(1): 91-104.

Grau, A. J., A. Reis, et al. (2001). "Monocyte function and plasma levels of interleukin-8 in acute ischemic stroke." J Neurol Sci $192(1-2)$ : 41-47.

Hadi, S. M., S. H. Bhat, et al. (2007). "Oxidative breakage of cellular DNA by plant polyphenols: a putative mechanism for anticancer properties." Semin Cancer Biol 17(5): 370-376.

Imamura, G., A. A. Bertelli, et al. (2002). "Pharmacological preconditioning with resveratrol: an insight with iNOS knockout mice." Am J Physiol Heart Circ Physiol 282(6): H1996-2003.

Johnston, S. C. (2004). "Ischemic preconditioning from transient ischemic attacks? Data from the Northern California TIA Study." Stroke 35(11 Suppl 1): 2680-2682.

Karanth, H. and R. S. Murthy (2007). "pH-sensitive liposomes-principle and application in cancer therapy." J Pharm Pharmacol 59(4): $469-483$.

Kharlamov, A., S. C. Jones, et al. (2002). "Suramin reduces infarct volume in a model of focal brain ischemia in rats." Exp Brain Res 147(3): 353359.

Lee, K. W., J. K. Kundu, et al. (2006). "Cocoa polyphenols inhibit phorbol ester-induced superoxide anion formation in cultured HL-60 cells and expression of cyclooxygenase-2 and activation of NF-kappaB and MAPKs in mouse skin in vivo." J Nutr 136(5): 1150-1155.

LePage, K. T., R. W. Dickey, et al. (2005). "On the use of neuro-2a neuroblastoma cells versus intact neurons in primary culture for neurotoxicity studies." Crit Rev Neurobiol 17(1): 27-50.

Liang, W., T. S. Levchenko, et al. (2004). "Encapsulation of ATP into liposomes by different methods: optimization of the procedure." J Microencapsul 21(3): 251-261.

Lieberthal, W., S. A. Menza, et al. (1998). "Graded ATP depletion can cause necrosis or apoptosis of cultured mouse proximal tubular cells." Am J Physiol 274(2 Pt 2): F315-327.

Mahesh, R., H. W. Jung, et al. (2011). "Joongpoongtang 05 (JP05) confers neuroprotection via anti-apoptotic activities in Neuro-2a cells during oxygen-glucose deprivation and reperfusion." Toxicol In Vitro 25(1): 177-184.

Millart, H., L. Alouane, et al. (2009). "Involvement of P2Y receptors in pyridoxal-5'-phosphate-induced cardiac preconditioning." Fundam Clin Pharmacol 23(3): 279-292.

Murzakhmetova, M., S. Moldakarimov, et al. (2008). "Antioxidant and prooxidant properties of a polyphenol-rich extract from Geranium sanguineum L. in vitro and in vivo." Phytother Res 22(6): 746-751.

Nemeikaite-Ceniene, A., A. Imbrasaite, et al. (2005). "Quantitative structure-activity relationships in prooxidant cytotoxicity of polyphenols: role of potential of phenoxyl radical/phenol redox couple." Arch Biochem Biophys 441(2): 182-190.

O'Collins, V. E., M. R. Macleod, et al. (2006). “1,026 experimental treatments in acute stroke.” Ann Neurol 59(3): 467-477.

Park, L., J. Anrather, et al. (2004). "Abeta-induced vascular oxidative stress and attenuation of functional hyperemia in mouse somatosensory cortex." J Cereb Blood Flow Metab 24(3): 334-342.

Online access at http://www.aestm.com/?p=817 
Raza, H. and A. John (2005). "Green tea polyphenol epigallocatechin-3-gallate differentially modulates oxidative stress in PC12 cell compartments." Toxicol Appl Pharmacol 207(3): 212-220.

Robaszkiewicz, A., A. Balcerczyk, et al. (2007). "Antioxidative and prooxidative effects of quercetin on A549 cells.” Cell Biol Int 31 (10): $1245-1250$.

Romero-Perez, A. I., R. M. Lamuela-Raventos, et al. (2001). "Method for the quantitative extraction of resveratrol and piceid isomers in grape berry skins. Effect of powdery mildew on the stilbene content." J Agric Food Chem 49(1): 210-215.

Saver, J. L., G. W. Albers, et al. (2009). "Stroke Therapy Academic Industry Roundtable (STAIR) recommendations for extended window acute stroke therapy trials." Stroke 40(7): 2594-2600.

Shinjyo, N. and K. Kita (2006). "Up-regulation of heme biosynthesis during differentiation of Neuro2a cells." J Biochem $139(3)$ : $373-381$.

Simonyi, A., Q. Wang, et al. (2005). "Polyphenols in cerebral ischemia: novel targets for neuroprotection.” Mol Neurobiol 31(1-3): 135-147.

Tremblay, R. G., M. Sikorska, et al. (2010). “Differentiation of mouse Neuro 2A cells into dopamine neurons.” J Neurosci Methods 186(1): 60-67.

Tsai, S. K., L. M. Hung, et al. (2007). "Resveratrol neuroprotective effects during focal cerebral ischemia injury via nitric oxide mechanism in rats." $\mathrm{J}$ Vasc Surg 46(2): 346-353.

Weimar, C., K. Kraywinkel, et al. (2002). "Etiology, duration, and prognosis of transient ischemic attacks: an analysis from the German Stroke Data Bank." Arch Neurol 59(10): 1584-1588.

Weintraub, M. I. (2006). "Thrombolysis (tissue plasminogen activator) in stroke: a medicolegal quagmire." Stroke 37(7): 1917-1922.

Yamaguchi, T., M. Nomura, et al. (2009). "Effects of frequency and power of ultrasound on the size reduction of liposome." Chem Phys Lipids 160(1): 58-62.

Yonekura, I., N. Kawahara, et al. (2004). “A model of global cerebral ischemia in C57 BL/6 mice.” J Cereb Blood Flow Metab 24(2): 151-158.

Zhao, J., N. Kobori, et al. (2006). "Sulforaphane reduces infarct volume following focal cerebral ischemia in rodents." Neurosci Lett 393(2-3): 108112.

Zhen, G. and S. Dore (2007). "Optimized protocol to reduce variable outcomes for the bilateral common carotid artery occlusion model in mice." $\mathrm{J}$ Neurosci Methods 166(1): 73-80.

\section{Funding Statement}

This work was supported by NIH grant 7R21NS065912-02

\section{Acknowledgements}

None 